\title{
SEMI-AUTOMATIC CATHETER MODEL GENERATION USING BIPLANE X-RAY IMAGES
}

\author{
Sebastian Kaeppler ${ }^{1 *} \quad$ Wen $W^{2} \quad$ Terrence Chen ${ }^{2} \quad$ Martin Koch ${ }^{1}$ \\ Atilla P. Kiraly ${ }^{2} \quad$ Norbert Strobel $^{3} \quad$ Joachim Hornegger ${ }^{1}$ \\ ${ }^{1}$ Pattern Recognition Lab, Friedrich-Alexander-Universität Erlangen-Nürnberg, Germany \\ ${ }^{2}$ Siemens Corporation, Corporate Technology, Princeton, NJ, USA \\ ${ }^{3}$ Siemens AG, Healthcare Sector, Forchheim, Germany
}

\begin{abstract}
Recently, techniques for the automatic detection or tracking of surgical instruments in X-ray guided computer-assisted interventions have emerged. The purposes of these methods are to facilitate either inter-modality registration, motion compensation, enhanced visualization or automatic landmark generation in augmented-reality applications. Most techniques incorporate a model of the device as prior information to evaluate results obtained from a low-level detector. In this paper, we present novel approaches which are able to generate both 2-D and 3-D models of circular and linear catheters from biplane X-ray images with only minimal user input. We apply these methods in the context of Electrophysiology to generate models of ablation and mapping catheters. An evaluation on clinical datas sets yielded promising results.
\end{abstract}

Index Terms - X-ray, Biplane, Ablation, Mapping, Catheter, Model, Semi-automatic, Reconstruction, Detection, Electrophysiology

\section{INTRODUCTION}

C-arm fluoroscopy is the standard imaging modality for cardiac catheterization procedures. In addition to the standard fluoroscopic images, pre- and intra-procedurally acquired volumetric images can be rendered on top of the 2-D images to improve navigation. During the last few years, automatic device detection and tracking methods for X-ray images have been proposed. These methods enable a variety of further applications and improvements, such as motion compensation [1, 2], intra-modality registration [3], enhanced visualization or automated landmark generation. Almost all methods are based on a common principle - low-level detection results are evaluated in the context of a model, representing the shape of the device that is to be detected. While the model can be fixed in the detection algorithm for some devices, the variety or adjustability of others requires a manual delineation of the device by the user to initialize the detection algorithm. However, increased user interaction might reduce the clinical

* Sebastian Kaeppler was with Siemens Corporation while conducting this work. acceptance of such methods. In order to overcome this disadvantage, we propose two novel semi-automatic algorithms for 2-D catheter detection and 3-D shape model generation using a biplane X-ray image pair. The first algorithm is capable of detecting and reconstructing flexible linear catheters, for example diagnostic or ablation catheters. The second algorithm is for circular catheters, such as the mapping or the pigtail catheter. The method for linear catheters requires only one click on the catheter body in one imaging plane. The method for circular catheters requires one click on the catheter top in each plane. Both methods are capable of generating 2-D and 3-D catheter models. Additionally, the position of electrodes on the catheter can be detected and reconstructed. The resulting 3-D models may not only be used to initialize detection algorithms, but also for 3-D visualization of the catheter. While our algorithms are designed for biplane images, they can easily be adapted to generate 2-D models from monoplane images. Compared to previous work on catheter detection and reconstruction $[4,5,6]$, our methods favor speed and robustness on challenging images over generality by including more prior knowledge of the catheter that is to be detected.

\section{METHODS}

We demonstrate the effectiveness of our algorithms by applying them to two catheter types commonly used in Electrophysiology (EP) procedures: ablation and circular mapping catheters. Note that our algorithms may easily be adjusted to other types of catheters by a change of parameters.

\subsection{Linear Ablation Catheter}

Ablation catheters are linear catheters with a diameter of about $2.4 \mathrm{~mm}$. The distal part of the catheter consists of a tip, and depending on type and manufacturer, two or three additional electrodes. This part is of interest for detection and visualization. We separate the catheter model generation process into four stages: Joint 2-D body detection, 2-D body refinement, 3-D model reconstruction and 3-D electrode detection. Initially, the user has to select a point on the catheter body in one of the image planes. A quadratic region of inter- 

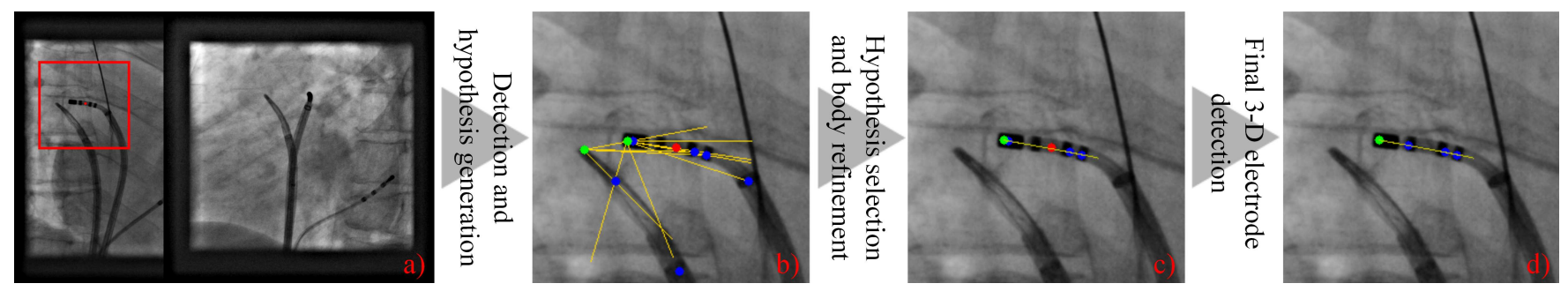

Fig. 1. Ablation catheter detection steps: (a) biplane input images, (b) detection results and body hypotheses, (c) refined body, (d) final result. The user click is depicted in red, the electrode and tip candidates in blue and green, respectively.

est (ROI) with a side length of $5 \mathrm{~cm}$ is centered around the click in the image plane (Fig. 1(a)). In the other image plane, the ROI is determined by the epipolar lines of top and bottom of the ROI that was determined in the first plane. In both planes, the ROI is further restricted by obtaining the positions of the shutter blades. As described in [2], we employ two probabilistic boosting tree classifiers with Haar-like features on the ROI. One is trained to detect the tip of the catheter, the other to detect electrodes. For catheters with only two additional electrodes, we treat the end of the tip as an electrode. Local maxima of the detection maps are found using non maximum suppression. We refer to these 2-D points as tip and electrode candidates and denote them as $t_{A}$ and $e_{A}$ for plane $\mathrm{A}$, and $t_{B}$ and $e_{B}$ for plane $\mathrm{B}$, respectively. For the tip, we keep two candidates with the highest probability in each plane, for the electrodes we keep six. Using these candidates, we perform a joint coarse detection of the catheter body in both planes by evaluating a set of hypotheses. Each hypothesis $H$ is determined by selecting a tip and an electrode candidate in each plane $\left(t_{A}^{H}, t_{B}^{H}, e_{A}^{H}, e_{B}^{H}\right)$. Hypotheses and detection candidates in one plane are depicted in Fig. 1(b). We exhaustively evaluate all 144 resulting hypotheses by a scoring function. The score $S(H)$ for hypothesis $H$ consists of a 3-D tip probability $T(H)$ and two 2-D body scores $B_{A}(H), B_{B}(H)$ and is computed as

$$
S(H)=T(H) \cdot\left(B_{A}(H)+B_{B}(H)\right) .
$$

The tip probability $T(H)$ is modeled by multiplying the 2-D detection probabilities of the tip candidates with each other and the probability of their correspondence

$$
T(H)=p\left(t_{A}^{H}\right) \cdot p\left(t_{B}^{H}\right) \cdot p_{\mathrm{cor}}\left(t_{A}^{H}, t_{B}^{H}\right) .
$$

The correspondence probability $p_{\text {cor }}\left(t_{A}^{H}, t_{B}^{H}\right)$ measures the likelihood of the correspondence of the two 2-D points based on the symmetric epipolar distance.

The body scores for each plane are computed by fitting a 2-D line, where the start point is the tip candidate, the direction is the vector from the tip candidate to the electrode candidate, and the length is a device- and geometry-dependent value. All tip and electrode candidates as well as the point supplied by the user contribute to the score of the body based on their detection probability and their orthogonal distance to this line. Candidates in one plane whose epipolar lines do not intersect with the hypothesis' line in the other plane do not contribute to the score.

After the scoring process, only the hypothesis with the highest score is kept. In each plane, a rectangle is positioned around the previously determined line and all electrode and tip candidates which are not within the rectangle are removed. The next step in the body detection process is the 2-D model refinement. A 2-D second degree polynomial curve is fitted to the detection candidates in each plane (Fig. 1(c)). The catheter tip is used as starting point, and the fitting is performed using weighted ridge regression, with the weights corresponding to the detection probabilities of the electrode candidates. Regularization is applied to the curve's curvature to yield a more robust fit.

The third step is 3-D body reconstruction. One of the polynomial curves is densely sampled. For each sample point, its epipolar line in the other image is computed and intersected with the other 2-D curve. Since we intersect a line with a 2-D second degree polynomial curve, we may get two solutions. The intersection point corresponding to the previously determined line model is selected. For each pair of corresponding points, a 3-D point is triangulated. The set of 3-D points represents the catheter body in 3-D. The reconstruction process is stopped after a 3-D model with a predetermined and catheter specific length (in this case, $17 \mathrm{~mm}$ ) has been reconstructed.

To detect the 3-D positions of the catheter electrodes, each 3 -D point is assigned a score which is the sum of the probabilities of its corresponding 2-D points. In this context, we use the maximum of the tip and electrode classifier probabilities as probability in 2-D. Afterwards the 3-D model is split up into segments with a length of $0.5 \mathrm{~mm}$, starting after the tip. For each segment, the 3-D point with the highest score is determined and stored as a 3-D electrode candidate. Different hypotheses for catheter electrode configurations are generated by selecting all combinations of three 3-D electrode candidates which satisfy physical constraints (minimum and maximum electrode distance, minimum catheter length). Each hypothesis is evaluated by another scoring function, which multiplies the scores of the three 3-D electrode candidates. Electrode candidates corresponding to a local maximum of the probability along the 3-D model boost the hypothesis 
score by factor two. The three 3-D electrode candidates of the hypothesis with the highest score are chosen as catheter electrodes. If necessary, the 3-D tip and electrode points can be forward projected to obtain 2-D electrode locations. The final result of our detection algorithm is shown in Fig. 1(d).

\subsection{Circular Mapping Catheter}

The mapping catheter consists of a circular tip that is positioned at the ostia of the Pulmonary Veins to measure electrical signals. Typical mapping catheters have ten to twenty electrodes. Our semi-automatic detection of its tip is based on a fast shape candidate generation-and-testing approach. Promising candidates are stored and optimized before a final hypothesis selection stage. The detection and reconstruction process is started with the user marking the top of the circular tip in both planes (Fig. 2(a)). Using these corresponding points, a 3-D point is triangulated. A rectangular ROI is positioned in each plane at the top of the catheter.

As before, a probabilistic boosting tree with Haar-like features is applied to detect electrode candidates $e_{A}, e_{B}$ in 2-D. The fifteen candidates with the strongest response are stored. Similar to the approach presented in [1], we also perform vesselness filtering of the image, followed by thresholding, skeletonization and distance map computation. However, we refine the veselness map after thresholding by removing all filter responses which are not connected to an electrode candidate (Fig. 2(b)).

Since the catheter tip is circular, we chose to represent it as a circle in 3-D, which can be parameterized with six parameters: 3-D center, radius and direction. Using the constraint that the 3-D point supplied by the user is the top of the circle, the center may be computed analytically for a given radius and direction. We uniformly sample radii and directions on a half sphere (due to the symmetricity of a circle) and use them compute the center of a 3-D circle hypothesis. In total we generate about 11000 hypotheses with ten different radii, ranging from 6.0 to $15.0 \mathrm{~mm}$. Thus, we sample about 1100 directions per radius. Each hypothesis $H$ is assigned a probability $p(H)$, which is computed as:

$$
p(H)=p_{A}(H) \cdot p_{B}(H) \cdot p_{\text {prior }}(H) \cdot p_{\text {const }}(H) .
$$

The prior term $p_{\text {prior }}(H)$ models prior knowledge about the radius of the catheter. Since the mapping catheter is positioned at the ostia of a pulmonary vein, we model it as a mixtures of gaussians, with parameters taken from medical literature [7]. The soft constraint term, $p_{\text {const }}(H)$, prevents solutions which are too far away from the points marked by the user. At this stage, this particular term has no effect since all hypotheses were created using the user supplied points as constraint.

The data terms $p_{A}(H)$ and $p_{B}(H)$ measure the fit of the hypothesis to the image in plane $\mathrm{A}$ and $\mathrm{B}$, respectively. We model each of these data probabilities by averaging two probabilistic sub-measurements. The first one measures how many of the detected electrode candidates are accounted for by the hypotheses. We reduce the detection probability of each electrode candidate based on its distance to the forward projected 3-D circle. The sum of all distance-adjusted probabilities is divided by the sum of the original probabilities and multiplied by a constant, yielding the first measurement. The second measurement of the fit of the circle hypothesis uses the distance map. We uniformly sample points on the 3-D circle and forward project them. The distance map is sampled on these points. Due to foreshortening, too many samples may fall on top and bottom of the resulting ellipse in 2-D. We employ a weighting scheme to counter this effect and compute a weighted average of the distance to the detected catheter body. The resulting value is mapped into a value range of $[0 . .1]$.

All hypotheses with a probability below a predetermined threshold are discarded. We chose the value for the thresholding such that typically 100 to 200 hypotheses remain. The parameters of the remaining hypotheses (one example is shown in Fig. 2(c)) are optimized by applying a limited number of iterations of the well known Broyden-FletcherGoldfarb-Shanno (BFGS) method to minimize the function

$$
p^{\prime}(H)=-\left[p_{A}^{\prime}(H) * p_{B}^{\prime}(H) * p_{\text {prior }}(H) * p_{\text {const }}^{\prime}(H)\right] .
$$

This function is a variant of Eq. (3). The soft constraint term is more relaxed and the data terms only consider the distance map. Omission of the electrode information leads to a smoother objective function which is less susceptible to get stuck in a local minimum. Essentially, this step may be considered as 2-D/3-D registration comparable to the approach presented in [1]. Afterwards, all hypotheses which have been optimized are evaluated a second time using Eq. (3). This step is justified, since Eq. (3) captures more information, while Eq. (4) is more suitable for parameter optimization. The hypothesis with the highest probability is selected as 3-D catheter model. 2-D models may easily be obtained by forward projecting the 3-D model, as depicted in Fig. 2(d). To obtain electrode positions in 2-D, the electrode candidate probabilities are first adjusted based on their distance to the 2-D model. The candidates with the highest probability are selected. 3-D coordinates of electrodes can be obtained by back projecting the 2-D electrode candidates onto the catheter model in 3-D.

\section{EXPERIMENTAL RESULTS}

\subsection{Evaluation workflow}

In our evaluation of the linear ablation catheter reconstruction, the second electrode of the catheter in plane A was marked and the reconstruction was started automatically hereafter. Since we had no ground truth 3-D model of the catheter available, we forward projected the detected tip and electrodes into both 2-D images. We measured the distance between the detected and manually annotated tip and 


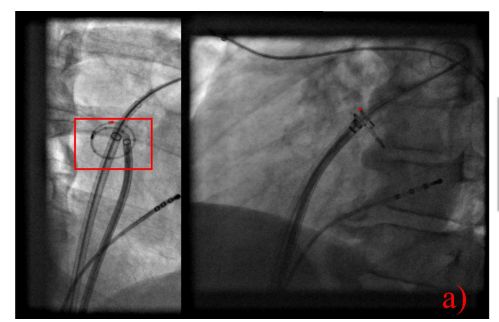

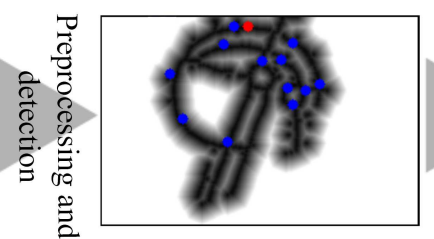

b)

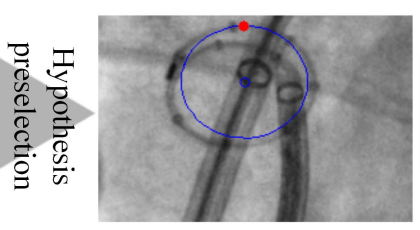

c)

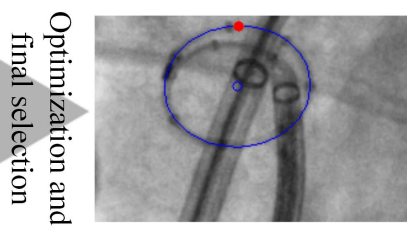

d)

Fig. 2. Mapping catheter detection steps: (a) biplane input images, (b) preprocessing results, (c) intermediate shape candidate, (d) final result. The user clicks are depicted in red, the electrode candidates in blue.

electrodes. If none of those distances exceeded $2 \mathrm{~mm}$, we categorized the detection as successful.

For the mapping catheter, the catheter top was marked in both planes and the reconstruction algorithm was started. We forward projected the detected 3-D model and measured the distance to the catheter body at the point of the highest distance. If the mapping catheter was not completely closed, i.e., if there was a gap in its circular tip, we ignored this gap. Our criterion of a successful detection was a maximum distance of $2 \mathrm{~mm}$.

\subsection{Evaluation results}

For our evaluation, we we used 46 biplane images to evaluate the ablation catheter reconstruction. For the mapping catheter reconstruction, we used 37 image pairs. The images were acquired in two clinics during live interventions. Applying the criterion described above resulted in a successful initialization rate of $91 \%(42 / 46)$ for the ablation and $92 \%(34 / 37)$ for the mapping catheter. On our test system (Intel Core i72720QM, 8GB RAM), the maximum runtime was $702 \mathrm{~ms}$ for the mapping, and $436 \mathrm{~ms}$ for the ablation catheter detection.

\section{CONCLUSION}

In this paper, we proposed two semi-automatic methods to detect and reconstruct linear and circular catheters in both 2-D and 3-D using a biplane X-ray image pair. An evaluation of both techniques using clinical data demonstrated their performance and robustness. Compared to previous work, the proposed methods incorporate more knowledge about the catheters that are to be detected. We believe that this information is necessary to achieve acceptable performance in challenging scenarios.

Our method to reconstruct linear catheters could easily be turned into a fully automatic catheter detection by removing the restriction to a region of interest and the user initialization term in the body selection. Initial results in this direction are promising. Additionally, both algorithms may easily be adapted to 2-D operation on single plane images by simply removing the data terms considering the second plane. We are also currently investigating how additional seed points can be integrated into the framework to correct failed detections.

\section{Acknowledgements:}

This work was supported by the German Federal Ministry of Education and Research (BMBF) in the context of the initiative Spitzencluster Medical Valley, project grant Nos. 01EX1012A and 01EX1012E. Additional funding was provided by Siemens AG, Healthcare Sector. The concepts and information presented in this paper are based on research and are not commercially available.

\section{REFERENCES}

[1] A. Brost, R. Liao, N. Strobel, and J. Hornegger, "Respiratory Motion Compensation by Model-Based Catheter Tracking during EP Procedures," Medical Image Analysis, vol. 14, no. 5, pp. 695-706, 2010.

[2] W. Wu, T. Chen, P. Wang, S. Zhou, D. Comaniciu, A. Barbu, and N. Strobel, "Learning-based hypothesis fusion for robust catheter tracking in 2D X-ray fluoroscopy," in CVPR. 2011, pp. 1097-1104, IEEE.

[3] A. Brost, F. Bourier, L. Yatziv, M. Koch, J. Hornegger, N. Strobel, and K. Kurzidim, "First Steps towards Initial Registration for Electrophysiology Procedures," in SPIE Medical Imaging, 2011, vol. 7964, 79641P (2011).

[4] M. Hoffmann, A. Brost, C. Jakob, F. Bourier, M. Koch, K. Kurzidim, J. Hornegger, and N. Strobel, "Semiautomatic Catheter Reconstruction from Two Views," in MICCAI. 2012, number 7511 in LNCS, pp. 584-591, Springer.

[5] E. Franken, P. Rongen, M. van Almsick, and B. ter Haar Romeny, "Detection of Electrophysiology Catheters in Noisy Fluoroscopy Images," in MICCAI. 2006, number 4191 in LNCS, pp. 25-32, Springer.

[6] H.-J Bender, R. Maenner, C. C. Poliwoda, S. Roth, and M. Walz, "Reconstruction of 3D catheter paths from 2D X-ray projections," in MICCAI. 1999, number 1679 in LNCS, pp. 981-989, Springer.

[7] Y.-H. Kim, E. Marom, J. Herndon, and H. McAdams, "Pulmonary vein diameter, cross-sectional area, and shape: CT analysis.," Radiology, vol. 235, no. 1, pp. 439; discussion 49-50, Apr 2005. 